\title{
生物玻璃/聚乳酸多孔微球的制备及其作为细胞载体的研究
}

\author{
高 龙 $^{1,2}$, 张赵文斌 ${ }^{1,2}$, 常 江 $^{1,2}$ \\ (1. 中国科学院 上海硅酸盐研究所, 上海 200050;2. 中国科学院大学, 北京 100049)
}

摘 要: 具有大孔结构的多孔微球既可以在体外扩增细胞, 还可以作为细胞的传输工具, 通过注射的方式把细胞输 送到需要修复的组织部位。生物玻璃虽然生物活性良好, 但难以直接制备成大孔结构的微载体。因此, 本研究将生 物玻璃(BG)与聚乳酸(PLA) 高分子复合, 通过复乳法制备了一种含生物玻璃的多孔微球细胞微载体。并通过扫描电 镜(SEM)、热重分析(TGA)、电感耦合等离子体发射光谱仪(ICP-OES)等方法研究分析了微球的形貌、组成和离子 释放。通过细胞实验，证明细胞可以在微球的多孔结构中粘附和增殖，并且生物玻璃可以促进细胞增殖，在组织工 程中具有潜在应用。

关 键 词: 多孔微球; 生物玻璃; 聚乳酸; 细胞微载体; 组织工程

中图分类号: R 318.08 文献标识码: A

\section{Bioglass/Polylactic Acid Porous Microspheres: Preparation and Their Application as Cell Microcarriers}

\author{
GAO Long $^{1,2}$, ZHANG Zhaowenbin ${ }^{1,2}$, CHANG Jiang ${ }^{1,2}$ \\ (1. Shanghai Institute of Ceramics, Chinese Academy of Sciences, Shanghai 200050, China; 2. University of Chinese Academy \\ of Sciences, Beijing 100049, China)
}

\begin{abstract}
Porous microsphere cell microcarrier with macroporous structure can not only amplify cells in vitro, but also serve as cell delivery tools to deliver cells to damaged tissues by injection. Bioglass (BG) is an inorganic material with excellent biological activity, however, it is difficult to directly prepare microcarriers with macroporous structure. Therefore, in this study, a BG/poly-lactic acid (PLA) porous microspheres was prepared by double emulsion method. The morphology and structure of the microsphere were characterized by SEM, and the load of BG in the microsphere was characterized by thermo gravimetric analysis and its ion release was detected by ICP. Cell proliferation experiments showed that cells could adhere and grow on the surface and inside of the microspheres. The results show that the microsphere with interconnected open-pore microstructure is suit for cell adhesion and proliferation. Bioglass can promote cell proliferation in the microspheres and the obtained BG/PLA composite microsphere has great potential applications in tissue engineering.
\end{abstract}

Key words: porous microsphere; bioglass; polylactic acid; cell microcarrier; tissue engineering

收稿日期：2019-11-23; 收到修改稿日期：2019-12-19

基金项目: 国家重点研发计划(2016YFC1100201); 中国科学院战略性先导科技专项(XDA16010203)

National Key Research and Development Plan of China (2016YFC1100201); The Strategic Priority Research Program of the Chinese Academy of Sciences (XDA16010203)

作者简介：高 龙(1988-), 男, 博士研究生. E-mail: gaolong@sina.cn GAO Long (1988-), male, PhD candidate. E-mail: gaolong@sina.cn

通讯作者：常 江，研究员. E-mail: jchang@mail.sic.ac.cn CHANG Jiang, professor. E-mail: jchang@mail.sic.ac.cn 
方法把细胞或药物输送到缺损部位 ${ }^{[3-4]}$ 。特别是具有 开放式大孔结构的微球, 既能够在体外培养时帮助 细胞向内生长, 又能在注射过程中起到支撑作用, 以保护微球内部的细胞免于挤压和摩擦 ${ }^{[5]}$ 。基于上 述的优异性能, 微球细胞微载体已经用于骨缺损修 复 ${ }^{[6-9]}$ 、软骨再生 ${ }^{[10-11]}$ 和心肌修复 ${ }^{[12]}$ 。但是, 多孔微 球细胞微载体往往缺乏生物活性, 在体外细胞扩增 或者体内细胞传输过程中, 材料本身没有促进细胞 增殖、迁移和分化的生物活性。通过添加生长因子 等方式获得生物活性, 往往也存在生长因子脆弱、 容易失活和价格昂贵等弊端。所以, 在组织修复当 中迫切需要一种具有良好生物活性的细胞微载体。

生物玻璃作为一种无机材料, 不仅在骨和牙齿 的修复领域应用广泛 ${ }^{[13-15]}$, 也被证实在软组织修复 中具有良好的生物活性 ${ }^{[16]}$ 。但是, 生物玻璃难以直 接制备成微球细胞微载体。首先, 生物玻璃难以塑型, 制备出来的微球没有细胞培养所需的大孔结构 ${ }^{[17]}$; 其次, 纯生物玻璃微球离子释放速率过快, 其 $\mathrm{Si}$ 离 子浓度甚至会超过 $60 \mu \mathrm{g} / \mathrm{mL}$, 过高的离子浓度可对 细胞增值有抑制作用, 比如会抑制成纤维细胞的迁 移和分化 ${ }^{[16]}$ 。此外, 生物玻璃快速的离子交换, 还 会造成过强的碱性环境, 产生细胞毒性。目前, 常见 的多孔微球细胞微载体往往是由高分子材料制备而 成的, 虽然具有良好的生物相容性和孔结构, 但是, 缺乏刺激细胞增殖和分化的生物活性。因此, 将生 物玻璃与高分子材料复合, 制备出一种既具有良好 生物活性, 又具有多孔结构的微球细胞微载体, 对 于组织缺损修复及组织工程应用具有重要意义。

因此, 本研究利用复乳法制备了一种生物玻 璃/聚乳酸复合微球细胞微载体。首先, 生物玻璃能 够释放活性离子, 促进细胞在微载体上的粘附和 增殖, 并且由于离子的浓度比纯生物玻璃微球低, 避免了离子浓度过高对细胞的抑制作用。其次, 聚 乳酸具有良好的可塑性, 可以制备出贯通的多孔 结构, 适宜细胞在其表面和内部的粘附和增殖。同 时, 聚乳酸材料具有弱酸性, 生物玻璃呈碱性, 与 聚乳酸复合可以避免聚乳酸降解产生的酸性对细 胞产生不利影响。在本研究当中, 详细探讨了聚乳 酸浓度和致孔剂用量对微球结构的影响。同时, 将 复合微载体用于细胞的体外扩增, 考察了细胞在 微球细胞微载体表面及内部孔结构上的粘附以及 细胞的活力。

\section{1 实验方法}

实验所使用的聚乳酸(PLA), 分子量为 100 万,
购于济南岱罡生物工程有限公司。生物玻璃, 型号 为 $45 \mathrm{~S} 5$, 购于昆山华侨科技新材料有限公司。二氯 甲烷、明胶和聚乙烯醇(PVA)统一购置于国药集团化 学试剂有限公司。

\subsection{BG/PLA 微球的制备}

BG/PLA 微球的制备过程如图 1 所示:

首先, 将一定量 PLA加入到 $20 \mathrm{~mL}$ 二氯甲烷中, 充分溶解后, 加入 $0.2 \mathrm{~g}$ 生物玻璃和一定量的致孔剂 (6.5\%的明胶水溶液)。利用高速剪切乳化机, 在 $15000 \mathrm{r} / \mathrm{min}$ 的条件下, 将上述混合液快速乳化, 得 到乳液备用。

然后, 将上述乳液加入到 $50 \mathrm{~mL}$ 预冷至 $2{ }^{\circ} \mathrm{C}$ 的 $0.1 \% \mathrm{PVA}$ 溶液中。在 $800 \mathrm{r} / \mathrm{min}$ 的速率下磁力搅拌 $2 \mathrm{~min}$, 将乳液分散成小球。

最后, 将上述小球收集在 $800 \mathrm{~mL}$ 预冷至 $2{ }^{\circ} \mathrm{C}$ 的 $0.1 \% \mathrm{PVA}$ 溶液中, 通风梪内挥发去除溶剂。并用 大量 $50{ }^{\circ} \mathrm{C}$ 的清水洗去明胶, 获得多孔微球。

\section{2 表征分析}

将制备好的微球样品冷冻干燥好, 置于导电胶 上，喷金后，用扫描电子显微镜(JEM-2100F) 观察其 形貌。通过热重分析仪(TA-Q500), 分析复合微球中 的生物玻璃含量, 升温速率为 $10{ }^{\circ} \mathrm{C} / \mathrm{min}$, 最高温度 是 $700{ }^{\circ} \mathrm{C}$, 气氛条件是氮气气氛。 $\mathrm{Si}$ 离子释放的测 试方法: 将制备的 $1.0 \mathrm{~g}$ 复合微球置于 $5 \mathrm{~mL}$ 去离子 水中, 在固定的时间点抽取上清液, 然后利用电感 耦合等离子体发射光谱仪(ICP-OES) 获得浸提液中 $\mathrm{Si}$ 离子的浓度。

\section{3 细胞的增殖}

本实验所用细胞为人骨髓间充质干细胞 (hBMSCs, Cyagen Biosciences)。在 $37{ }^{\circ} \mathrm{C}$ 恒温培养 箱, $5 \mathrm{vol} \% \mathrm{CO}_{2}$ 的湿润环境中培养, 每 $3 \mathrm{~d}$ 换一次培 养液。

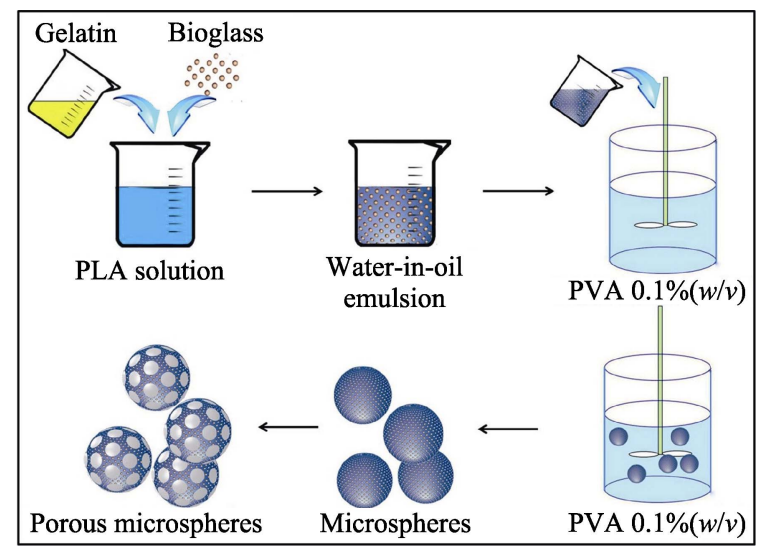

图 1 生物玻璃/聚乳酸复合微球细胞微载体制备示意图 Fig. 1 Schematic illustration for the preparation of BG/PLA composite microsphere cell microcarriers 
细胞在微球上接种方法如下。100 mL 培养血中, 将 $5 \times 10^{6}$ 个细胞与 $1 \mathrm{~mL}$ 微球进行混合。然后, 加入 $10 \mathrm{~mL}$ 培养基, 在培养箱中以 $75 \mathrm{r} / \mathrm{min}$ 转速振摇 $6 \mathrm{~h}$ 。最后, 加入 $20 \mathrm{~mL}$ 培养液转移至 $37{ }^{\circ} \mathrm{C} 、 5 \mathrm{vol} \%$ $\mathrm{CO}_{2}$ 培养箱内长期培养, 两天更换一次培养液。到 达预设时间 1、3 和 $7 \mathrm{~d}$ 时, 采用 Cell Counting Kit-8 (CCK8, Japan)试剂盒检测细胞活性。用 Live-dead 考察微球上的细胞分布以及生存和死亡情况。

\section{2 结果与讨论}

\subsection{BG/PLA 微球的制备}

粒径是评价微球细胞微载体的一个重要指标。 粒径过小, 不利于细胞的粘附; 粒径过大, 不利于 注射。研究表明, 直径约 $200 \mu \mathrm{m}$ 的微球细胞微载体 能够同时满足细胞粘附和注射的需求 ${ }^{[1]}$ 。PLA 浓度 是决定粒径的重要因素, 因此本课题组首先对聚乳 酸溶液的浓度进行了篮选。

从图 2 可以看出, 随着 PLA 溶液浓度的增加, 所得到的微球的平均直径越来越大。在 PLA 浓度为 $10 \%$ 的时候，所制备的微球平均直径约 $200 \mu \mathrm{m}$, 同 时分布在 $(200 \pm 20) \mu \mathrm{m}$ 范围内的微球比例最大, 约 为 $52 \%$ 。因此, 选取 $10 \%$ 的 PLA 溶液用于后续的微 球制备。

对于微球细胞微载体来说, 开放的多孔结构
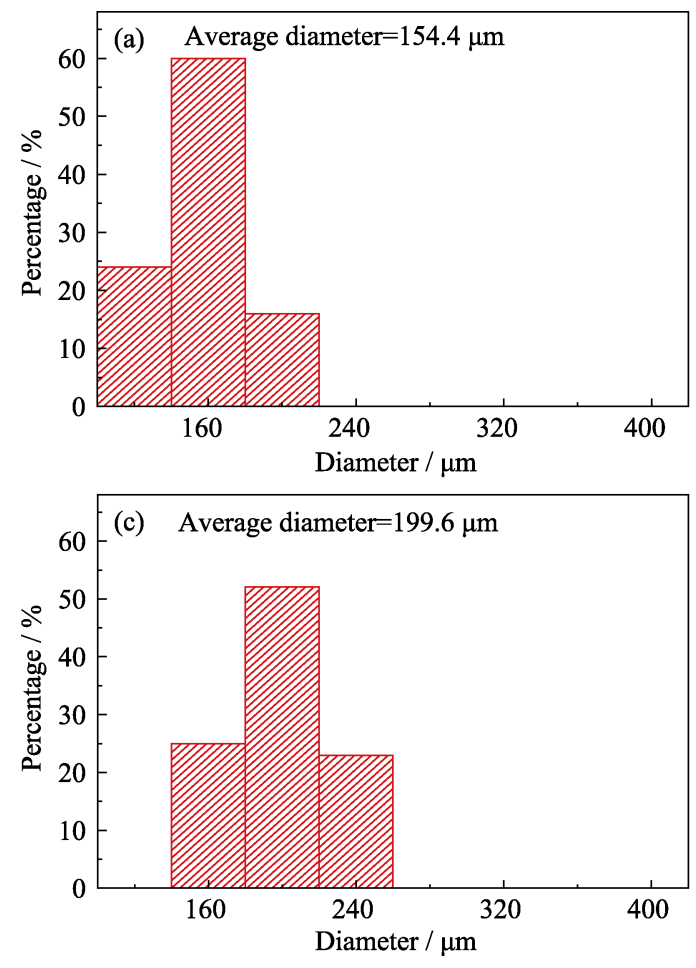

既有利于细胞自由地进出, 也适于氧气和营养物 质的传输以及代谢废物的排出。之前的研究表明, 直径 30 50 $\mu \mathrm{m}$ 的大孔, 最适于细胞进入微球内部 进行粘附和增殖 ${ }^{[1,10-11]}$ 。通过调节致孔剂用量, 制 备出不同孔结构的 BG/PLA 微球, 结果如图 3 所 示。通过微球的 SEM 照片可以看出, 随着致孔剂 含量的增加, 微球的孔径越来越大, 内部孔径之间 的贯通性也越来越好。但是, 当致孔剂含量达到 $8.0 \%$ 时，孔结构过大，会破坏微球的球形结构。而 致孔剂用量为 $6.0 \%$ 时, 所制备的微球既能维持大 量 $30 \sim 50 \mu \mathrm{m}$ 的大孔, 微球也能维持完整的结构, 是最适宜的用量。

如图 4 所示, 对孔径的进一步定量统计结果也 表明, 随着致孔剂含量的增加, 微球上孔的平均孔 径也会依次增加。当致孔剂的用量为 $6.0 \%$ 时, 微球 上直径 30 50 $\mu \mathrm{m}$ 的大孔比例最高，约为 7\%。同时， 大量小孔的同时存在也有助于营养物质的传输和代 谢废物的排出。因此，本课题组选取 $6.0 \%$ 致孔剂用 量用于后续的微球制备。

上述结果表明, 通过篮选适宜的 PLA 浓度和致 孔剂用量, 可以通过复乳法制备出粒径和孔结构都 适宜的多孔微球, 满足体外细胞三维培养对微载体 结构的要求。这一方法解决了生物玻璃难以塑型的 难题, 为生物玻璃材料在组织工程中的应用提供了 一种新的形式。
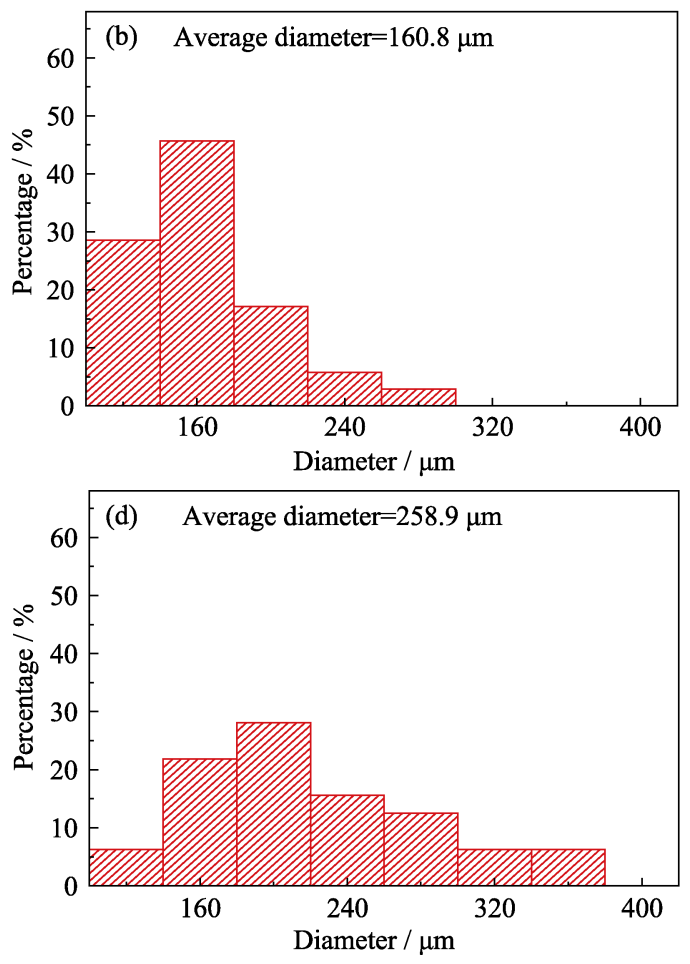

图 2 PLA 浓度为(a) $5.0 \%$ 、(b) $7.5 \%$ 、(c) $10.0 \%$ 、(d) $12.5 \%$ 的 BG/PLA 微球的粒径分布

Fig. 2 Particle size distribution of BG/PLA microspheres prepared with PLA solution concentration of (a) $5.0 \%$, (b) $7.5 \%$, (c) $10.0 \%$, and (d) $12.5 \%$ 

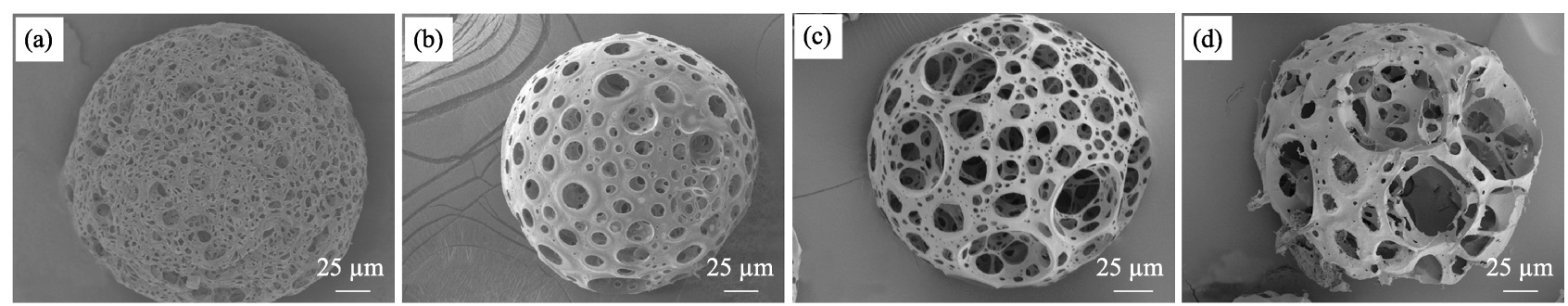

图 3 致孔剂用量为(a) $2.0 \%$ 、(b) $4.0 \%$ 、(c) $6.0 \%$ 、(d) $8.0 \%$ 的 BG/PLA 多孔微球

Fig. 3 BG/PLA porous microspheres prepared with (a) $2.0 \%$, (b) $4.0 \%$, (c) $6.0 \%$, and (d) $8.0 \%$ pore-forming agent
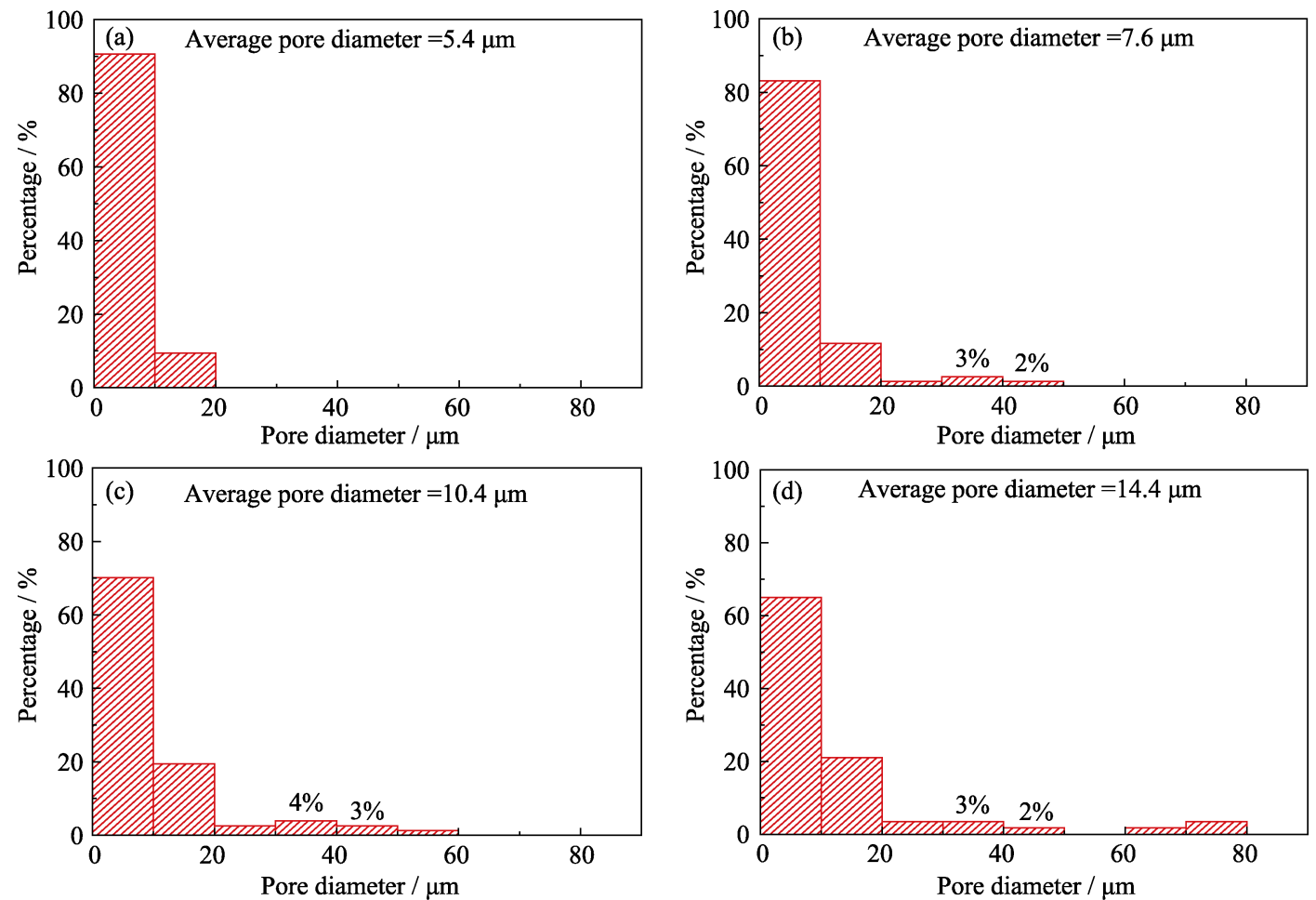

图 4 致孔剂用量为(a) 2.0、(b) 4.0、(c) 6.0、(d) $8.0 \%$ 的 BG/PLA 微球的孔径分布

Fig. 4 Pore size distribution of BG/PLA microspheres prepared with (a) 2.0, (b) 4.0, (c) 6.0 and (d) 8.0\% pore-forming agent

\section{2 复合微球中生物玻璃的含量及 $\mathrm{Si}$ 离子 释放}

本课题组通过复乳法得到了具有多孔结构的微 球，但是，单纯通过 SEM 照片还无法确定生物玻璃 颗粒是否复合在微球当中。生物玻璃主要通过释放 活性 $\mathrm{Si}$ 离子来发挥其促进细胞增殖、迁移和分化的 生物活性。因此, 首先通过热重分析, 来确定微球是 否含有生物玻璃。

结果如图 5 所示, 纯 PLA 微球在温度升高以后, 其有机高分子 PLA 会迅速降解和挥发, 在 $700{ }^{\circ} \mathrm{C}$ 时, 残余的质量几乎为零。而无机的生物玻璃在加热以 后, 质量变化很小, 在 $700{ }^{\circ} \mathrm{C}$ 时, 残余的质量约为 最初质量的 $96.2 \%$ 。在 BG/PLA 复合微球中, 温度 升高以后, PLA 的降解挥发造成质量迅速下降, 但 是 $\mathrm{BG}$ 则使得质量不会降为 0 , 在 $700{ }^{\circ} \mathrm{C}$ 时, 残余质 量为初始质量的 $4.8 \%$ 。通过上述结果, 可以计算出,

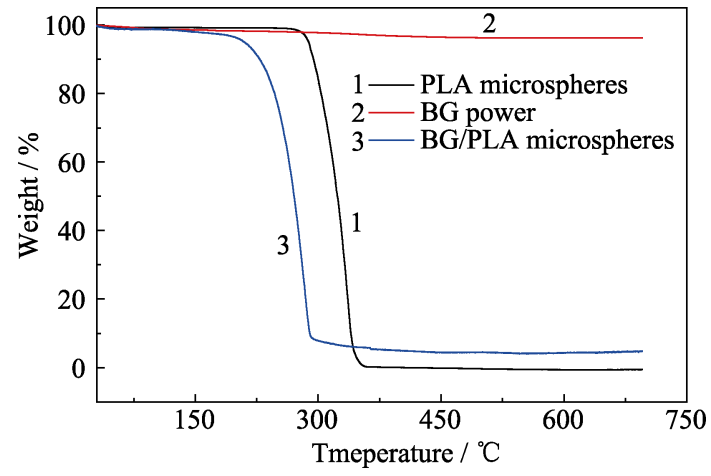

图 $5 \mathrm{BG} / \mathrm{PLA}$ 复合微球的热重分析图

Fig. 5 Thermo gravimetric analysis of BG/PLA microspheres

在复合微球当中聚乳酸的质量约为 $95.0 \%$, 生物玻 璃的质量约为 $5.0 \%$ 。

生物玻璃当中含有钠、钙、硅和磷等多种元素, 可以释放多种离子。但是，其促进细胞增殖、迁移 等主要是通过释放活性 $\mathrm{Si}$ 离子来实现的 ${ }^{[13,18]}$ 。因此, 
本课题组对复合微球活性 $\mathrm{Si}$ 离子的释放进行了测 定。结果如图 6 所示, BG/PLA 复合微球可以稳定释 放 $\mathrm{Si}$ 离子, 浓度在 1.2 2.2 $\mu \mathrm{g} / \mathrm{mL}$ 范围内。这说明, 生物玻璃与 PLA 复合以后，仍然可以通过释放活性 离子来发挥其生物活性。

上述的热重分析和离子释放结果进一步证明了 生物玻璃成功地复合到多孔微球当中, 并且仍可通 过释放 $\mathrm{Si}$ 离子来发挥其生物活性。同时，与之前报 道的纯生物玻璃微球相比, 其 $\mathrm{Si}$ 离子释放速率显著 降低，避免了浓度过高对于细胞的抑制，从而有利 于其发挥良好的生物活性。

\section{3 体外细胞实验}

为了考察引入生物玻璃对细胞在微载体上粘附 和增殖的影响, 本课题组分别用生物玻璃的 PLA 微 载体与 BG/PLA 微载体进行体外细胞扩增, 比较其 细胞粘附和增殖情况。

结果如图 7 所示, 在没有生物玻璃的 PLA 微载 体上, 细胞也可以进行粘附和增殖, 但是细胞的数 量相对较少, 并且多分布于微载体的表面, 进入内 部多孔结构的细胞数量较少。而在 BG/PLA 微载体 上, 不但细胞数量有了显著增加, 分布也更加广泛。 这说明生物玻璃的引入，增加了微载体扩增细胞的 效率。

此外, 本课题组还采用 CCK8 对细胞的活性进 行了定量分析。结果如图 8 所示, BG/PLA 微球上的 细胞比纯 PLA 微球上的细胞具有更高的活力。这说 明生物玻璃可以明显地提高细胞活性, 提高了细胞 体外扩增的效率。

细胞进入微球内部, 既可以拓宽增殖的空间, 又 能减少注射时细胞所受的伤害, 是评价微球细胞微载 体的重要指标 ${ }^{[5]}$ 。为了进一步研究细胞是否真正进入 BG/PLA 微球细胞微载体内部, 本课题组在微球上分 别选取不同深度的截面进行分析。如图 9 所示, 在 BG/PLA 复合微球细胞微载体的不同深度截面上

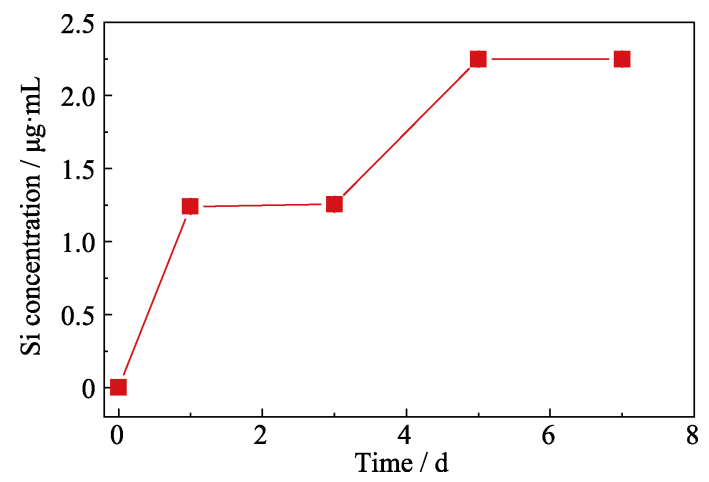

图 $6 \mathrm{BG} / \mathrm{PLA}$ 复合微球的 $\mathrm{Si}$ 离子释放

Fig. $6 \mathrm{Si}$ ions released from the BG/PLA microspheres
均有大量的细胞粘附, 而且存在细胞的面积与微球 相应的截面积相当, 说明细胞在微球细胞微载体三 维结构中粘附和增殖良好。
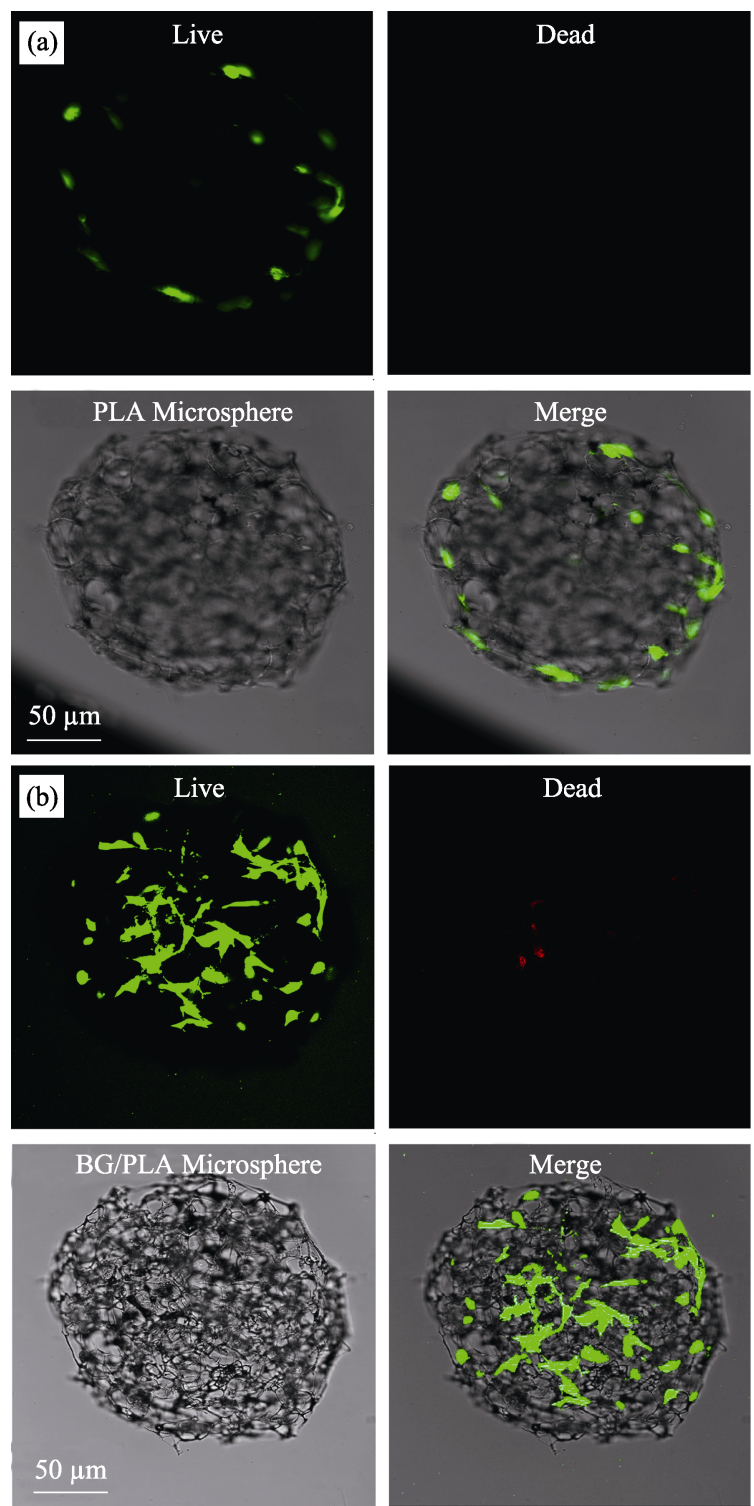

图 7 (a)PLA 微球和(b)BG/PLA 微球上细胞的活死染色照片 Fig. 7 Live-dead cell staining of cells on (a) PLA microspheres and (b) BG/PLA microspheres

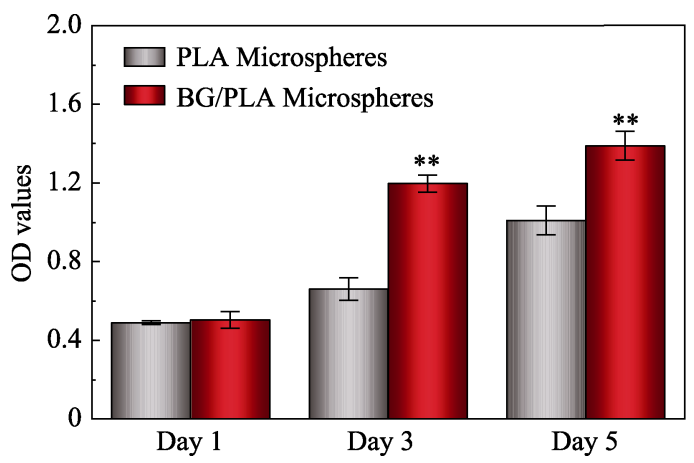

图 8 细胞在微球上的增殖

Fig. 8 Proliferation of cells on microspheres 

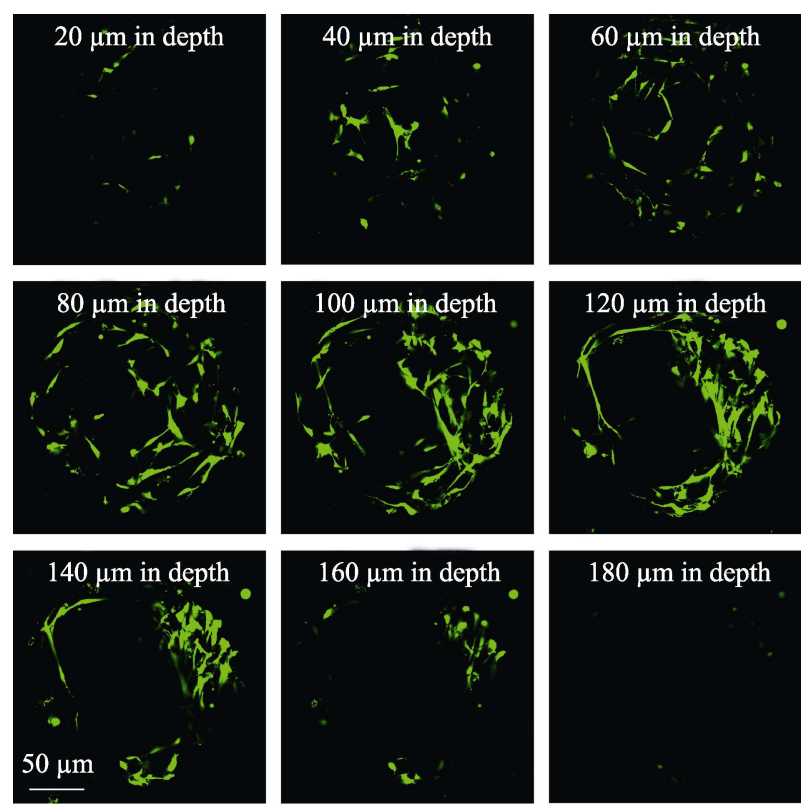

图 9 细胞在 BG/PLA 微球不同截面上的分布

Fig. 9 Distribution of cells on different cross sections of BG/ PLA microspheres

体外细胞实验结果表明, BG/PLA 微球能够有 效地促进细胞的扩增。通过释放活性 $\mathrm{Si}$ 离子, $\mathrm{BG} /$ PLA 微球贯穿的大孔结构提供了更大的空间, 从而 能够快速扩张细胞, 满足组织工程的需求。同时, 微 球的刚性结构和活性离子还可以为进入其内部的细 胞提供良好的保护作用, 避免其在后续的注射过程 中受到摩擦损伤。对于细胞在生物组织中的驻留, 更好地修复受损组织具有重要意义。

\section{3 结论}

1) 采用复乳法将生物玻璃与聚乳酸复合, 能 够获得具有适宜直径和贯通大孔结构的复合微球。

2) BG/PLA 复合微球细胞微载体能够稳定地释 放活性 $\mathrm{Si}$ 离子，促进细胞在其表面的粘附和增殖。

3) 细胞既可以在 BG/PLA 复合微球的表面增殖, 还可以进入其大孔内部，大大提高了细胞扩增的效率。

4) 本研究所制备的复合微球细胞微载体既能 够体外扩增细胞, 又具有合适的粒径满足注射的需 求, 在组织工程领域具有广阔的应用前景。

本研究通过细胞体外实验初步验证了 BG/PLA 复合微球在促进细胞粘附及增殖方面的 优势。但仍有一系列科学问题有待进一步研究, 比如: 细胞进入到 BG/PLA 微球内部结构以后, 这一特殊的离子和结构环境对细胞有何影响; BG/PLA 复合微球释放的活性硅离子能否促进细 胞在体内组织中的驻留和定向分化等。通过系统 的体内外生物学实验来回答这些科学问题以后,
可以进一步实现 $B G / P L A$ 复合微球在骨、心肌、 脂肪等组织修复中的应用。

\section{参考文献:}

[1] MALDA J, FRONDOZA CG. Microcarriers in the engineering of cartilage and bone. Trends in Biotechnology, 2006, 24(7): 299-304.

[2] R. LANGER, J.P. VACANTI. Tissue engineering, Science. 1993, 260(5110): 920-926.

[3] ZHOU L, KONG J, ZHUANG Y, et al. Ex vivo expansion of bone marrow mesenchymal stem cells using microcarrier beads in a stirred bioreactor Biotechnology and Bioprocess Engineering, 2013, 18(1): 173-184.

[4] QIU QQ, DUCHEYNE P, AYYASWAMY PS. New bioactive, degradable composite microspheres as tissue engineering substrates. Journal of Biomedical Materials Research, 2000, 52(1): 66-76.

[5] WEI DX, DAO JW, CHEN GQ. A Micro-Ark for Cells: Highly Open Porous Polyhydroxyalkanoate Microspheres as Injectable Scaffolds for Tissue Regeneration. Advanced Materials, 2018, 30(31): 1802273

[6] BOO L, SELVARATNAM L, TAI CC, et al. Expansion and preservation of multipotentiality of rabbit bone-marrow derived mesenchymal stem cells in dextran-based microcarrier spin culture. Journal of Materials Science-Materials in Medicine, 2011, 22(5): 1343-1356.

[7] SU K, GONG Y, WANG C, WANG DA. A novel shell-structure cell microcarrier (SSCM) for cell transplantation and bone regeneration medicine. Pharmaceutical Research, 2011, 28(6): 14311441.

[8] CHUN KW, YOO HS, YOON JJ, et al. Biodegradable PLGA microcarriers for injectable delivery of chondrocytes: Effect of surface modification on cell attachment and function. Biotechnology Progress, 2004, 20(6): 1797-1801.

[9] YAN S, XIA P, XU S, et al. Nanocomposite porous microcarriers based on Strontium-substituted HA-g-poly (gamma-benzyl-1glutamate) for bone tissue engineering ACS Applied Materials \& Interfaces, 2018, 10(19): 16270-16281.

[10] FANG J, YONG Q, ZHANG K, et al. Novel injectable porous poly ( $\gamma$-benzyl-1-glutamate) microspheres for cartilage tissue engineering: preparation and evaluation. Journal of Materials Chemistry B, 2015, 3(6): 1020-1031.

[11] FANG J, ZHANG Y, YAN S, et al. Poly (L-glutamic acid)/chitosan polyelectrolyte complex porous microspheres as cell microcarriers for cartilage regeneration. Acta Biomaterialia, 2014, 10(1): 276-288.

[12] HUANG CC, WEI HJ, YEH YC, et al. Injectable PLGA porous beads cellularized by hAFSCs for cellular cardiomyoplasty. Biomaterials, 2012, 33(16): 4069-4077.

[13] HENCHLL, XYNOS I, EDGARA, et al. Preparation and biomineralization research of ultrafine sol-gel bioactive glass power. Journal of Inorganic Materials, 2002, 17(5): 897-909.

[14] DING FENG, LI NAN, LI YONG-SHENG, et al. Preparation of hierarchically porous MBG/CPC scaffold and its performance on drug loading and release. Journal of Inorganic Materials, 2013, 28(1): $97-102$

[15] ZHOU YAN-LING, FENG XIN-XING, ZHAI WAN-YIN. Study on the loading and releasing behavior of epirubicin hydrochloride from mesoporous bioactive glass (MBGs). Journal of Inorganic Materials, 2011, 26(1): 68-72.

[16] MIGUEZ-PACHECO V, HENCH LL, BOCCACCINI AR. Bioactive glasses beyond bone and teeth: emerging applications in contact with soft tissues Acta Biomaterialia, 2015, 13(1): 1-15.

[17] MIAO G, CHEN X, DONG H, et al. Investigation of emulsified, acid and acid-alkali catalyzed mesoporous bioactive glass microspheres for bone regeneration and drug delivery. Materials Science \& Engineering C-Materials for Biological Applications, 2013, 33(7): 4236-4243.

[18] BRAUER DS. Bioactive glasses-structure and properties Angewandte Chemie International Edition, 2015, 54(14): 4160-4181. 